\title{
Speckle pattern optimization for DIC technologies
}

\author{
Sz. Szalai1, ${ }^{1,}$, G. Dogossy ${ }^{2}$ \\ ${ }^{1}$ Széchenyi István University, Department of Vehicle Manufacturing, \\ Egyetem tér 1, 9026 Győr, Hungary \\ ${ }^{2}$ Széchenyi István University, Department of Materials Science and \\ Engineering, Egyetem tér 1, 9026 Győr, Hungary \\ *e-mail: szalaisz@sze.hu
}

Submitted: 11/11/2020; Accepted: 08/04/2021; Published online: 26/04/2021

Abstract: This paper contains the relation between speckle pattern and Digital Image Correlation (DIC). The most important advance in experimental mechanics has been DIC since the strain gage. The deformation (strain) of an object can be visualized by DIC. Among all scientific fields, the DIC Technologies have seen a dynamic increase. The relationship between the paint and the sample - as the patterns mediate the deformation to the cameras - has been the most important technological issue. In this article the method developed for the detection of isolated particles in alloys is used to characterize the spots, which help the best speckle pattern has determined.

Keywords: speckle pattern; digital image correlation (DIC); paint; airbrush

\section{Introduction}

Several methods are known from the literature for measuring deformations, displacements and stresses. Traditional measurement methods include, but are not limited to, strain gauges and inductive transmitters. These measurement methods require a lot of preparation and the use of expensive tools and instruments. The Digital Image Correlation (DIC) method used in my research, on the other hand, is a non-contact method that can be used to examine a complete displacement and elongation field in the parts of the specimen visible to the camera using ordinary tools. It is not necessary to know the location of the failure in advance for these measurements, as the measurement results apply to the entire specimen and are 
processed afterwards. Digital image correlation as a test procedure was first developed by a research team at the University of South Carolina in the 1980s and then developed continuously, but the dynamic leap began in the 2000s in parallel with the technological advancement of cameras, as shown in Fig. 1. The technology is not only emerging in the field of testing metallic materials, but is now also being used in the testing of polymeric, composite or even biological materials. In addition to conventional specimens, it has gained ground in complex components, machines, or even building components, or in biomechanics and medicine [1, 2, 3, 4].

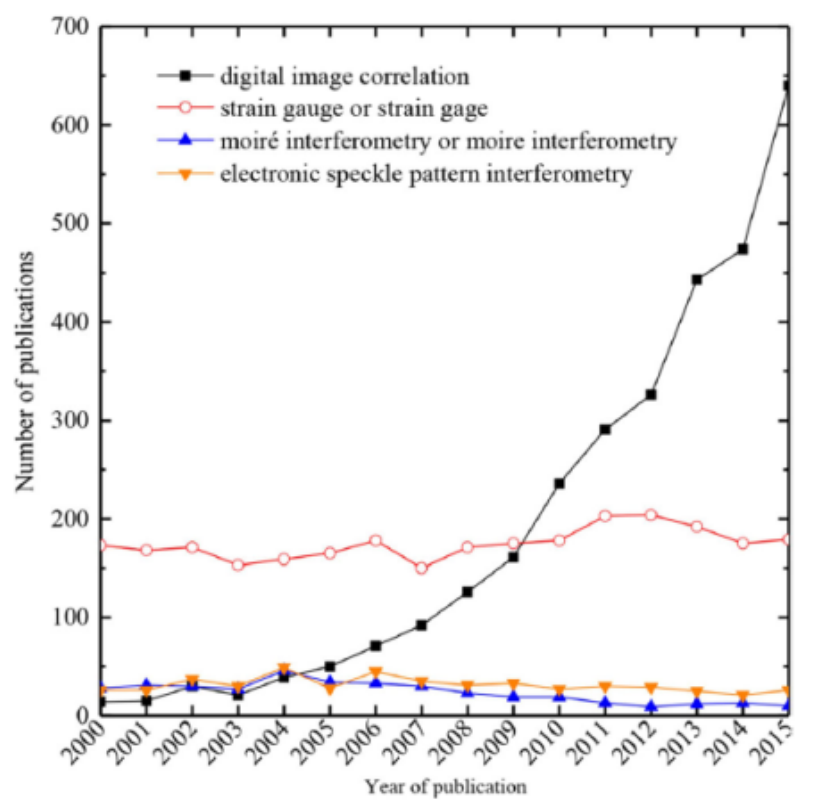

Figure 1. DIC growth in scientific articles [3]

According to the literature, the measurement of shape, displacement or deformation by the DIC technique requires the following:

- $\quad$ making a pattern - This pattern is placed on the surface of the examined objects and mediates the deformation of the object, in fact it has a kind of information-carrying role.

- $\quad$ taking pictures (photos) - The pattern and thus the specimen are recorded in different states, if we record with one camera we are talking about 2D DIC, in case of two cameras we can talk about stereo DIC or 3D DIC. 
- $\quad$ image analysis - After capture, the distorted images are compared to a reference or initial image using a special cross-correlation algorithm that will extract the displacement and stress fields [3].

It follows from the above and several other researches that one of the essential conditions for DIC measurements is the appropriate pattern (preferably random), which conveys the deformation information and greatly influences the fit of the images and the accuracy of the measurements.

When characterizing the patterns, the literature provides more aspects than an important research aspect. The speckle size should be determined from the relationship between the camera resolution used during image digitization and the measured area. From these two quantities, the pixel size in $\mathrm{mm}$ can be calculated, from which the literature indicates the optimal speckle size between 3-5 pixels [6, 7, 8]. Determining contrast is also an important factor in mapping (Fig. 2/a). A good contrast can be achieved with white spots on a black background or black spots on a white background to aid identification. For any patterned image, increase the contrast and reduce the noise. The best way to do this is a good paint and proper lighting [9, 10, 11]. Aliasing is also an important feature of the image (Fig. 2/b). Overlapping speckle points should be avoided at all costs. Any overlapping point increases the noise of the pattern, which compromises the accuracy of the measurement $[12,13,14]$.

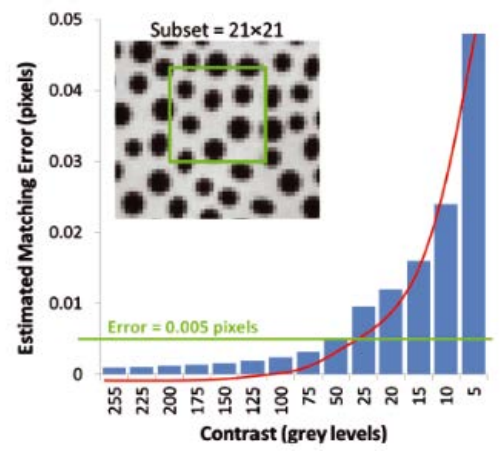

a)

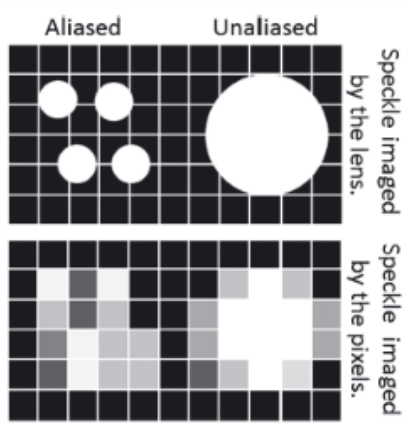

b)

Figure 2. Effect of contrast (a) and aliasing (b) $[8,15]$

In terms of determining contrast gradient, practice shows that it is always better to optimize the contrast, the size of the spots, and the degree of distribution at the cost of the sharpness of the edge of the spots. This is because traditional patterning methods often have sharp boundaries at the edges of the patterns, but this is difficult to accomplish with spray technologies. The traditional pattering methods include the 
raster technique, laser marking, dot painting, stenciling and dot stamping, which results a well-defined contour with good contrast. However, these techniques do not provide adequate randomness and richness of detail. The spot density is also basic requirement. The different traditional and spraying patterns can be seen in Fig. 3. Although it is difficult to make such a uniform pattern that would cause an erroneous hit, it is worthwhile to make the pattern as random as possible [15, 16, 17].

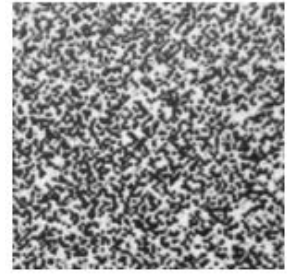

a)

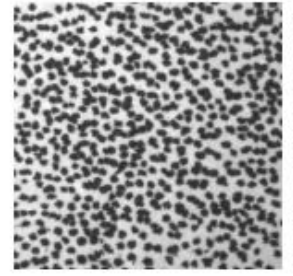

b)

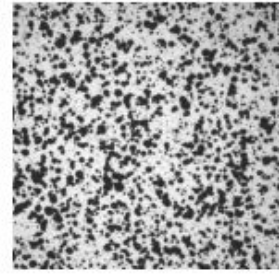

c)

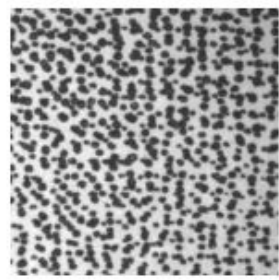

d)

Figure 3. Patterns of different densities and layouts [7]

The pattern of DIC techniques can be prepared by several methods as can be seen in the literature. The easiest and most accessible method of making patterns is spraying. In this case, a white base coat must be blown onto the specimen, on which the matte black diffused mottled pattern is applied (whether it is white on a black background or, conversely, it does not matter from the DIC point of view, the point is contrast). There are also several methods for applying stains. The following technique has proven to be useful for larger specimens and can be used quickly during most experiments: the specimen is sprayed by barely pressing the sprayer button. The propellant gas escaping slowly from the bottle will then not be able to completely disperse the dye, so droplets of ideal size will fall on the specimen. Another common procedure is to create a pattern with an airbrush gun, where you can better control the nozzle diameter and pressure, resulting in a much finer pattern and more reproducible results with the settings you set $[3,5,19,20]$.

For large specimens, stenciling can result in a similarly good pattern as in the case of a spray gun. However, electrolytic stenciling requires more expertise and cannot be applied to all materials. It is recommended to prepare medium or large products by printing. The pattern can also be applied to the surface with a laser or inkjet printer. Patterning with ink felt is also a possible solution. With this technique, quite large deformations can be measured, but it is very time consuming to apply. They can also be used directly on the surface, but it is also worth applying a white base coat here to increase the contrast. The grid pattern can be used among some certain restrictions, according to the literature it is a necessary rather than an optimal solution. Unlike the grid pattern, it is not a random pattern that allows for 
comparison, but in a raster design, the points are predetermined (intersection points). In the images, the points are not followed by searching for individual patterns, but by identifying each intersection point of the grid. This also results in an error in the method, as the raster may be so perfectly designed that one intersection point can be confused with another intersection point. However, due to the unevenness of the felt and the inhomogeneity of the surface to be examined, the areas are typically unique enough for continuous tracking. Some materials, such as wood veneer, have their own pattern, which, if the pattern parameters are appropriate, can be used for DIC measurements. If the material to be examined has a characteristic texture, it can still be used, but illumination can cause different shading on the textures, which makes two-camera measurements significantly more difficult and therefore not common [3, $5,19,20]$.

In addition to describing the various technologies and construction methods, it is important to clarify a few basic things about paints. The main components of paints are pigments, which give the color of the paint, binders, thinners and special materials. The paint can be water-based or oil-based. Water-based paints generally take less time to dry and cure than oil-based paints. Acrylic is also a popular type of paint. Acrylic paint is a paint emulsion consisting of pigments and plastic particles. The binder for the particles is a modified acrylic resin. The advantage of these paints is that they can be applied to almost any surface. They cure extremely quickly, usually in 2-3 hours, and today acrylic paints are water-based and thus water-soluble when wet. In the DIC technique, based on literature data and own experience, the following properties of paint should be used [3, 19]:

- matt gloss - should not flicker during the measurement,

- flexible - must follow the deformation of the specimen,

- only harden completely after more than 24 hours, because of the persistence of flexibility,

- $\quad$ easy to spray - preferably do not dilute as it also degrades the spectrum,

- deep black color - contains good quality pigment,

- $\quad$ water-based - this is more favorable for indoor applications [18].

It is clear from the list that these are often contradictory aspects, so it is difficult to find the right paint for the right job, and the composition of the paints can vary from country to country due to different regulations.

The literature classifies the parameters of a painting experiment according to several aspects, it is important that these parameters can only be set properly for airbrush (Fig. 4):

- dilution ratio,

- airflow or nozzle opening/size, 
- spreading distance,

- operating pressure $[3,6,18]$.

Fixed plate

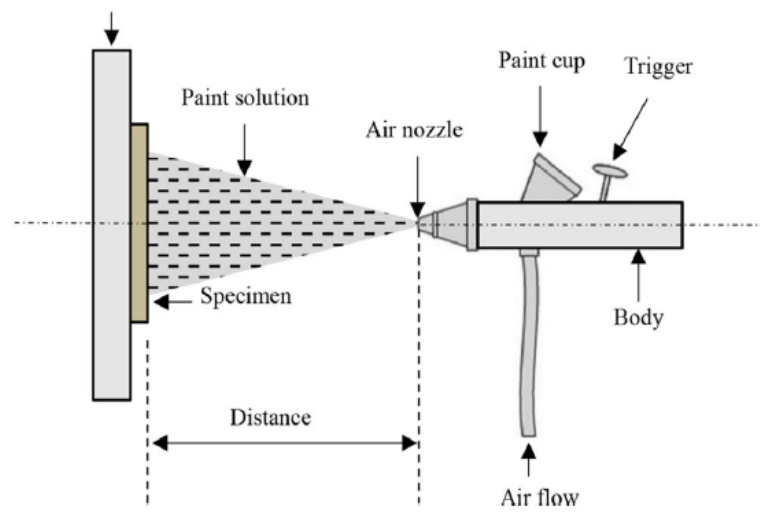

Figure 4. Airbrush set-up [3]

It is clear from the literature review that the difficulty of the characterization of the patterns stem from the technological production of the patterns on the one hand and the preparatory phase of DIC studies on the other hand. This article intends to place more emphasis on the appropriate pattern making technology, the pattern qualification process is discussed only in detail [21, 22, 23].

\section{Applied materials and methods}

There is no established method for characterizing the patterns, so a new, more comprehensive evaluation was developed than before. As a basic technique (Réti's window technique [24]), the method developed for the detection of isolated particles in alloys to characterize the spots was used. The patterns made with different technologies with the patterns issued and accepted by GOM was compared with the help of the method developed by the implementation of Réti's window technique [24]. Réti's window technique is used in metallography application to determine particulate rates. In the comparison, it was examined which painting technology can be used to achieve a nearly similar pattern.

During the research different types of black color paints were used:

1. water-based high quality lacquer spray, matte finish,

2. graphite-based graphite spray, matte finish,

3. acryl lacquer acryl spray, matte finish,

4. enamel paint, silky finish, 
5. elastic rubber paint, silky finish,

6. water-based enamel paint, matte finish.

The images were taken with Zeiss Axio Imager M1 optical stereo microscope with AxioVision 4.8 software and 2.5x optics. The tensile tests were carried out by Instron 5582 universal testing machine with $10 \mathrm{kN}$ and $100 \mathrm{kN}$ load cells, and Bluehill 2 software according to EN ISO 6892-1:2016 standard. The GOM ARAMIS 5M was used as DIC camera system with $800 \mathrm{~mm}$ adjustable base and GOM ARAMIS software. The Zeiss optical length measuring microscope with $1 \mu \mathrm{m}$ accuracy was used in validation. The Iwata HP-SP Plus adjustable nozzle airbrush pistol with oilfree compressor was used as airbrush system. The GOM ATOS Triple Scan II, with ATOS 2019 software was used as optical surface digitalization system. The Mahr PMC 800 coordinate measuring machine with MarSoft Vision 3D 4.1 software was used in validation. During the experiment Mitutoyo Absolute Digimatic ID-SX 543$781 \mathrm{~B}$ was used as dial indicator and Testo $174 \mathrm{H}$ calibrated digital temperature and humidity meter as thermometer. The used equipment can be seen in Fig. 5.

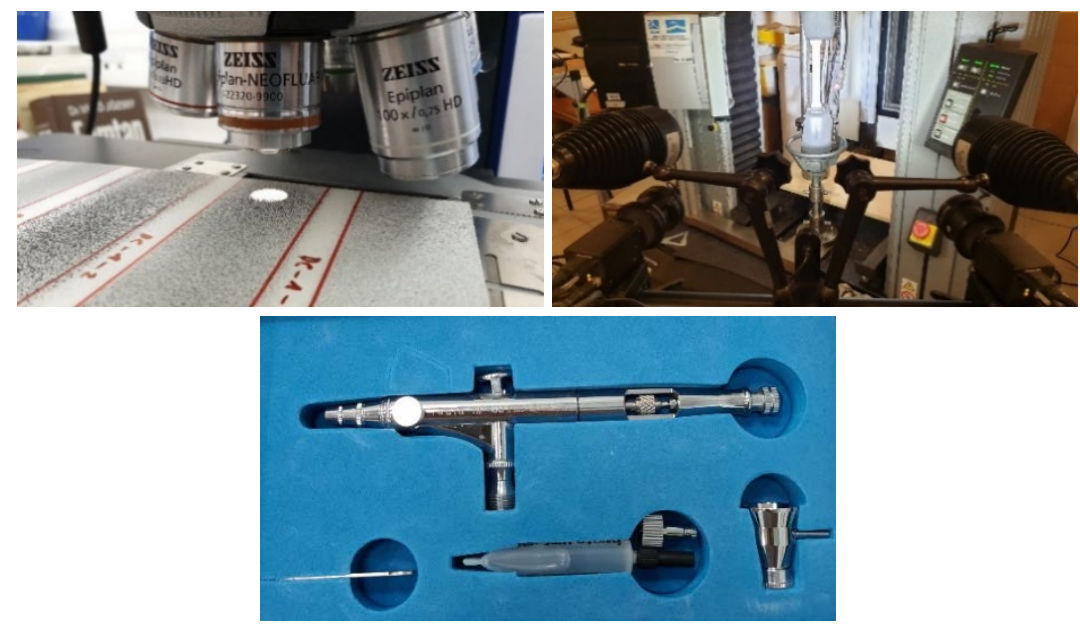

Figure 5. Zeiss Optical Stereo Microscope (a) and ARAMIS 5M test setup with tensile testing machine (b) and Iwata AirBrush pistol (c)

The specimen was made from AlMg3 alloy (thickness of $1.5 \mathrm{~mm}$ ) and was produced with water cutting machine and complied with the dimensions specified in the standard (MSZ EN ISO 6892-1:2020). The specimen width was $20 \mathrm{~mm}$, measuring length was $120 \mathrm{~mm}$ and the total length was $250 \mathrm{~mm}$. 


\section{Experiment and Results}

The validation of the ARAMIS measurements process and the evaluation of the different types of paints will be presented in this chapter. In addition to the results the patterns - which produced with different paints - will be described.

\subsection{Validation of the measuring system}

In the first step the measuring result of the ARAMIS system was verified. During this study the result of ARAMIS thickness reduction was compared with the result of ATOS, Mahr PCM 800 coordinate measuring machine and Mitutoyo dial indicator. Two of the three traditional measurements are tactile, while the ATOS and ARAMIS measures optically. Each system was calibrated and certified. The following figure (Fig. 6) shows the measurement results of ATOS and ARAMIS systems. The specimen was a 200x200x2.5 mm AlMg3 sheet for Nakazima test, it has been formed by a $100 \mathrm{~mm}$ diameter sphere until the crack.

During the validation process, the thickness of the formed plate was measured along the longitudinal axis of the specimen. The thickness was also measured with a dial indicator, a coordinate measuring machine and the ATOS system. These results were compared with the results of the ARAMIS system. The ATOS is also a coordinate measurement procedure, so it was interesting to compare it with traditional thickness measurement methods. The ARAMIS 5M system measures the major and minor strain on the surface (with the help of the painted pattern) and from this calculates the thickness reduction. The accuracy of the measurements is largely determined by the painted pattern, therefore the GOM calibration pattern was used for validation. It can be seen from the measurement results that next to $1.5 \mathrm{~mm}$ sheet thickness the measured results remained below 3\% difference (Table 1). A comparison of the ATOS and ARAMIS systems shows that the thickness reduction curves are also similar (Table 1 and Fig. 6). This test was verified by the thickness reduction measured with ARAMIS system.

Table 1. Thickness measuring results

\begin{tabular}{|l|c|c|}
\hline Measuring method & $\begin{array}{c}\text { Thickness next to } \\
\text { the crack [mm] }\end{array}$ & $\begin{array}{c}\text { Thickness on the top } \\
\text { of the specimen [mm] }\end{array}$ \\
\hline Dial indicator & 1.37 & 1.63 \\
\hline $\begin{array}{l}\text { Coordinate } \\
\text { measuring machine }\end{array}$ & 1.381 & 1.632 \\
\hline ATOS system & 1.39 & 1.65 \\
\hline ARAMIS system & 1.41 & 1.64 \\
\hline
\end{tabular}




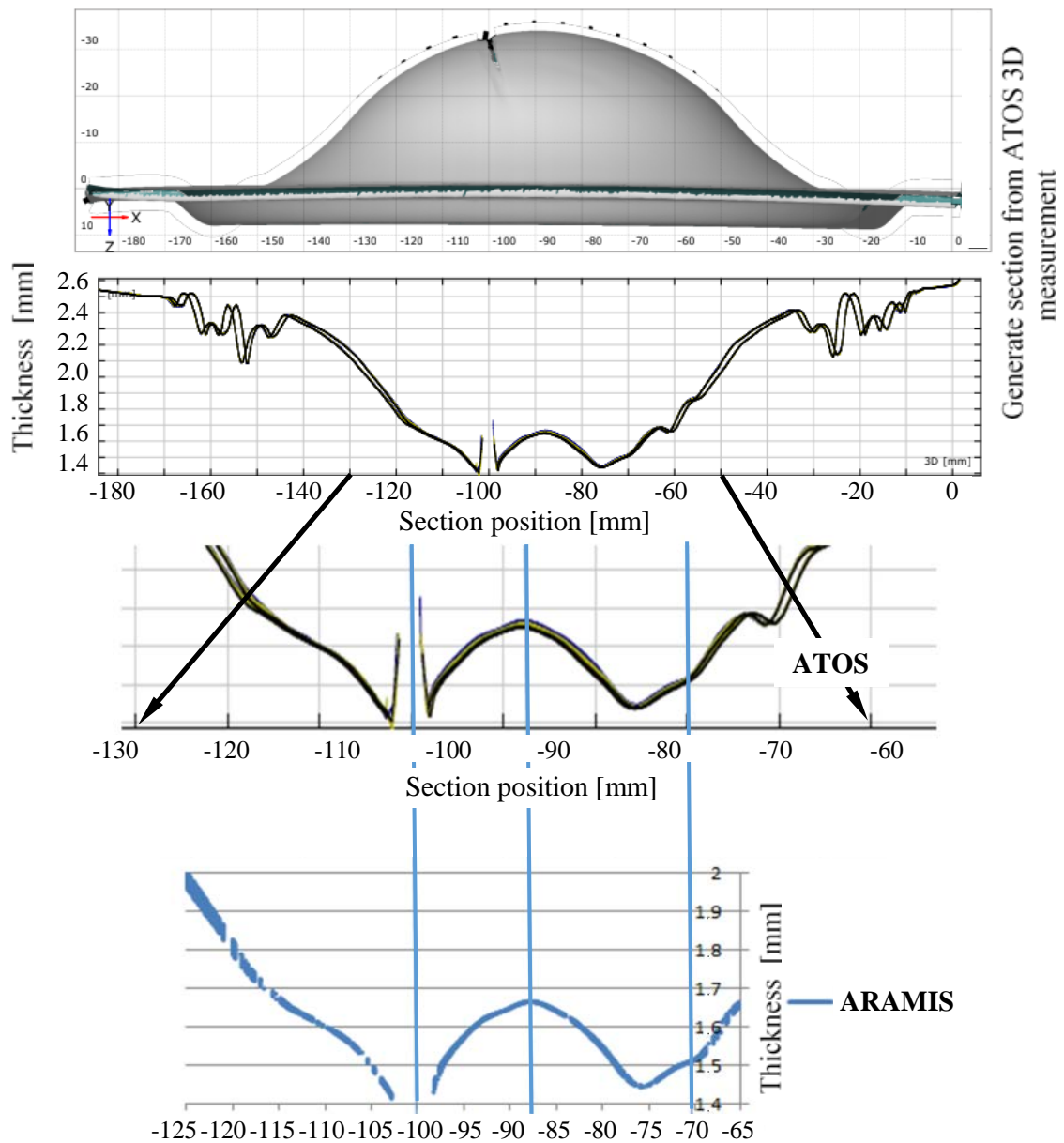

Section position [mm]

Figure 6. Validation of ARAMIS 5M system with thickness reduction

The aim of the research is to determine the appropriate patterns and staining parameters for ARAMIS systems. Whereas the ARAMIS system is a real-time 2D or 3D deformation measurement system, it is more important to verify the "online" elongation results when measuring the "static" thickness reduction. The tensile test is a 2D (from a metrological aspects) measurement process with good approximation, so validation is easier to perform with this test. Another major aspect 
is that the elongation measurement is important to determine the formability properties of the sheet. The next step was to validate this.

The AlMg3 validation specimens was $20 \mathrm{~mm}$ width and $1.5 \mathrm{~mm}$ thickness, the signal distance was $80 \mathrm{~mm}$, the total test section was $120 \mathrm{~mm}$ and the clamp width was $30 \mathrm{~mm}$. On the back of the specimens was made a Vickers imprint with load of $98.1 \mathrm{~N}$ at distance of $50 \mathrm{~mm}$. This distance was measured in an unformed state with an optical length measuring microscope with $0.001 \mathrm{~mm}$ accuracy. The specimens were formed in an Instron 5582 tensile testing machine with $5 \mathrm{~mm}$ crosshead displacement at speed of $5 \mathrm{~mm} / \mathrm{min}$. The DIC analysis of the specimens was performed with an ARAMIS 5M measuring system.

The tensile testing machine was stopped after $5 \mathrm{~mm}$ displacement, then the force was decreased to 0 , after then the samples were removed. The unloaded specimen was also recorded, so the whole process of the deformation history was recorded. In every $5 \mathrm{~mm}$ displacement from 5 to $25 \mathrm{~mm}$ the measure was stopped, this method 5 related DIC measurement and normal elongation measurement were recorded from each specimen. The results can be seen on Fig. 7.

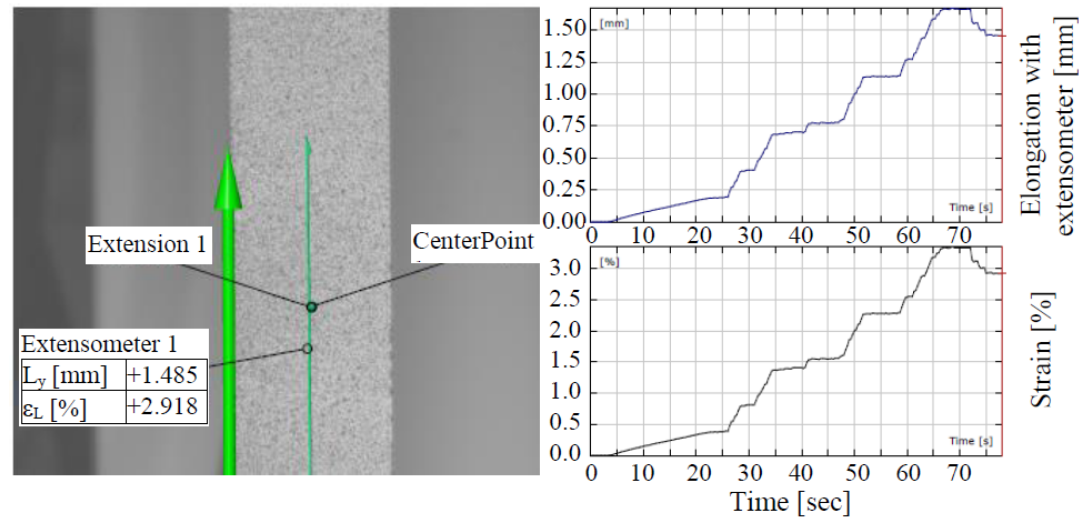

Figure 7. Virtual extensometer and extensometer results in ARAMIS software

After each increment the GOM sets the virtual extensometer to $50 \mathrm{~mm}$ and compares the $\Delta \mathrm{L}_{\mathrm{i}}$ increment in $\mathrm{mm}$ and the engineering strain in \%. The optical measuring microscope added the next increase to the raised deformation, so the elongation can be calculated from these data. The cumulative value of the increments was calculated during the evaluation, so the engineering strain were given in logarithmic strain as they can be summed. 


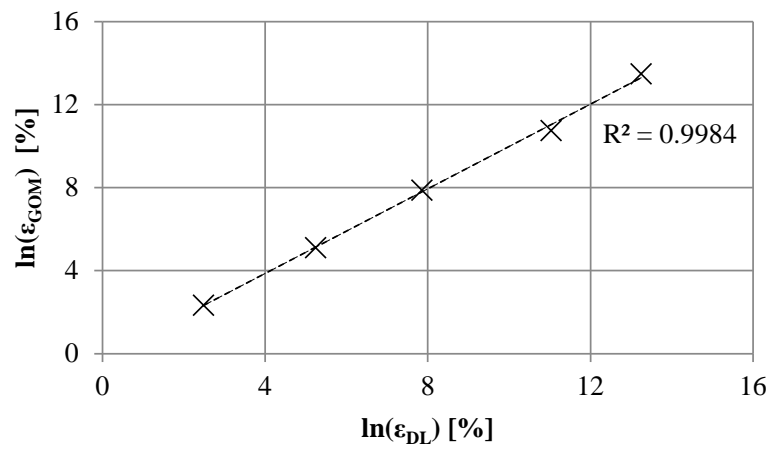

Figure 8. ARAMIS system validation with extensometer

The slope correlation coefficient diagram for all specimens is shown in Fig. 8. It can be seen that the coefficient of determination of the linear regression line of the measuring points is 0.9984 . With the uncertainty of this measuring system this is a very good result.

\subsection{Presentation of speckle painting experiments}

After the validation process different paints and painting technologies were tested. Spraying had to be done in such a way that the finished speckle pattern was as close as possible to the GOM calibrated pattern image. This is important so that the pattern error has the least effect on the measurement results, so only the effect of different painting methods and paints was measured. The different painting specimens were also compared to this. The adhesion of the primer layer was also critical. This layer transmits the deformation of the specimen to the cameras. If the adhesion of this layer is not good, then not the sheet deformation will be measured [7-15]. Surface preparation is also critical, so the specimen surface was cleaned with isopropanol alcohol. The primer layer testing process is not described in this article, but each specimen was dried 12 hours long at $22{ }^{\circ} \mathrm{C}$ and $40 \mathrm{RH} \%$ humidity. Measurements were made within 24 hours in all cases to avoid overdrying of the primer layer.

The painting was done with all 6 paints using (described in Section 2) airbrush and spray technology. The prepared patterns were checked and analyzed on an optical microscope. The corresponding patterns were compared with the GOM calibration image by visual inspection, with the AxioVision 4.8 software particle analysis and with the modified window technique (described in Section 2). In this measurement the particle size, contrast, distribution, pattern density, and particle dimension was measured. The patterns were also tested with the validation measurement process (elongation validation) to determine the acceptable patterns. 
The measurements showed that paint types 3 and 4 are not suitable for the test as they were extremely shiny. That is very important because the excessive reflection cannot be measured with the ARAMIS system. The glare is difficult to measure and it also distorts the measurement results. Their contrast is not adequate either, they were grey rather than black. Due to the properties of the type 5 elastic rubber paint, it cannot be applied with airbrush technology and it was also extremely shiny. These paints could not be used for further tests or measurements. The result can be seen on the Fig. 9.

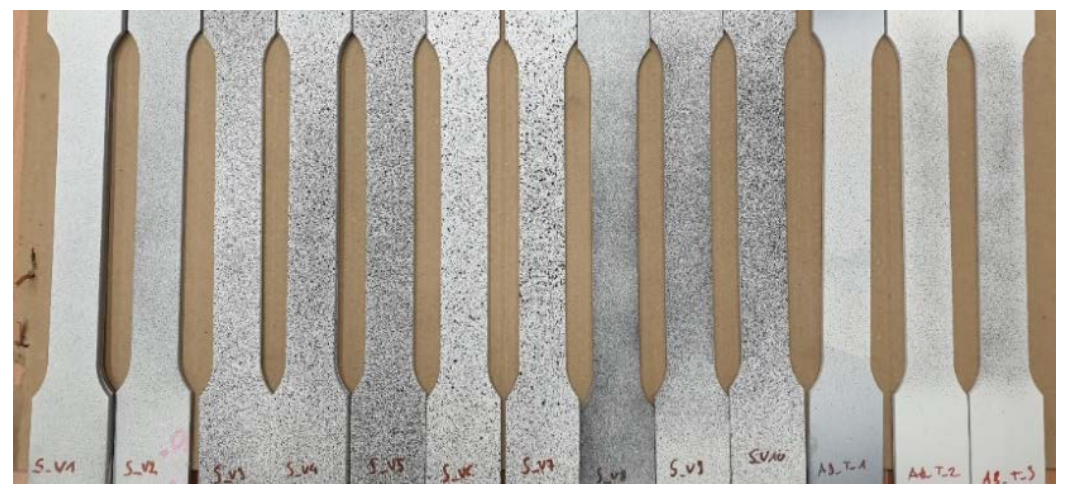

Figure 9. Specimens made with different paints and different techniques

The type 1, 2 and 6 paints were well suited and had good reflection. These results are the outcome of visual inspection. Further tests were made with these materials. In the next phase of the research the speckle patterns were analyzed. The coverage, the speckle area, average diameter, speckle number were measured for each specimen (Fig. 10). The results of the specimens were also compared with the results of the elongation measurements. Specimens where the error of the elongation measurement dropped below $1.5 \%$ were selected as acceptable patterns (Table 2). The $1.5 \%$ limit is derived from literature data. 

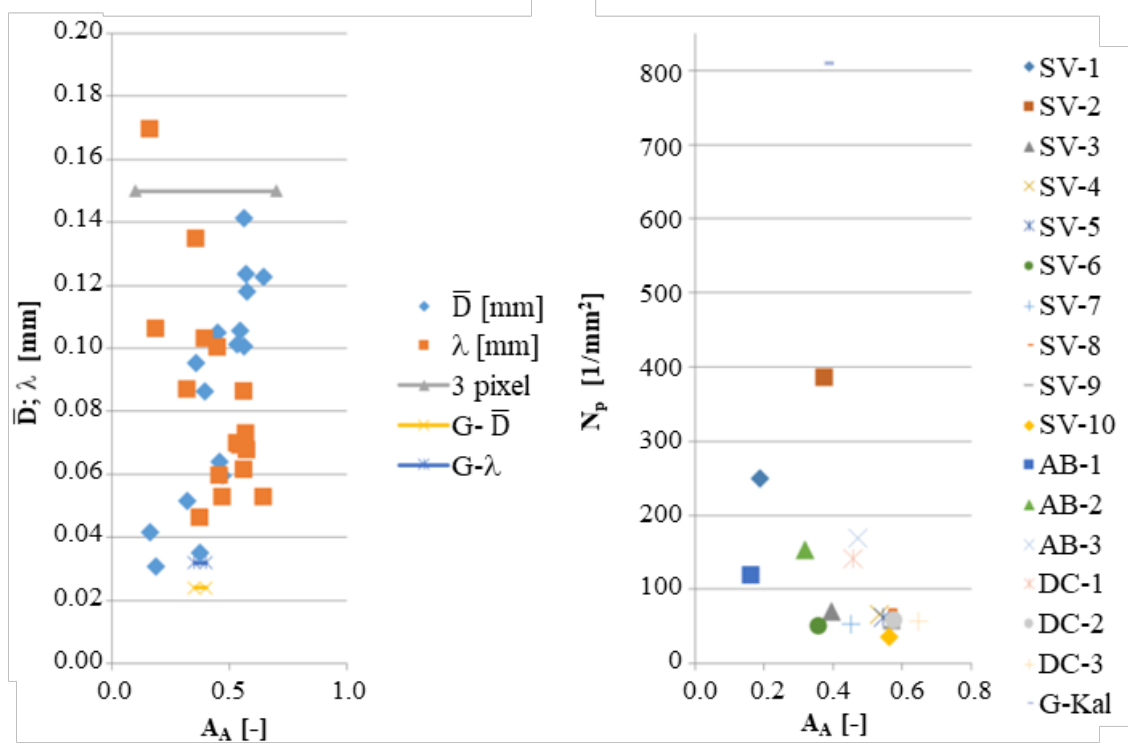

Figure 10. Comparison of patterns

Table 2. Samples with an error of less than $1.5 \%$ as a function of parameters (SV and $A B$ was the name of the specimen on Fig. 9)

\begin{tabular}{|l|l|l|l|l|l|l|l|}
\hline ABS(H\%) $<$ 1.5 & SV-1 & SV-2 & SV-3 & SV-5 & AB-1 & AB-2 & AB-3 \\
\hline $\mathbf{N}_{\mathbf{p}} \mathbf{5 0 - 2 5 0}$ & SV-1 & & SV-3 & SV-5 & AB-1 & AB-2 & AB-3 \\
\hline $\mathbf{A}_{\mathbf{A}} \mathbf{0 . 2 - 0 . 5}$ & SV-1 & SV-2 & SV-3 & & & AB-2 & AB-3 \\
\hline
\end{tabular}

An increase in the pattern aliasing and the average diameter of the speckles increases the error, while the number of speckle per $1 \mathrm{~mm}^{2}$ and the number of incisions in $1 \mathrm{~mm}$ decreases the failure. Based on the analysis of the approximate functions, it can be stated that in the middle of the ranges shown, the error is reduced to an acceptable level of about $1 \%$. Based on these, the parameters of the most favorable pattern can be determined which were also achieved with type 1, 2, 6 water based and graphite paints. Since these paints can be used with both spray and airbrush techniques, good results can be obtained by setting the painting parameters correctly. The usage of spray technique is faster but requires experience and better dexterity, while the usage of airbrush technology is slower, but when set up well does not require much professional knowledge. 


\section{Conclusion}

It was found in the analysis of the literature that the speckle patterns play critical role in DIC measurements. In the first phase of research the ARAMIS 5M DIC system was validated in two ways. In the second phase of research the different paints and paint spraying technologies were examined. It was determined that the best speckle pattern for DIC elongation tests has the following parameters: $0.3 \ldots 0.4$ coverage, $100 \ldots 2001 / \mathrm{mm}^{2}$ speckle number and $0.06 \ldots 0.10 \mathrm{~mm}$ average diameter. As a consequence, these above-mentioned parameters can be achieved by the waterbased high-quality matte finish lacquer spray, or the graphite-based matte finish spray, at $22^{\circ} \mathrm{C}$ and $40 \mathrm{RH} \%$ environment conditions. This research will be continued with various spraying techniques under different environmental conditions.

\section{References}

[1] M. A. Sutton, J. J. Orteu et al., Introduction to digital image correlation: Best practices and applications, Experimental Techniques 36 (1) (2012) pp. 3-4. doi: $h$ ttps ://doi .org/10.1111/j.1747-1567.2011.00798.x

[2] Y. L. Dong, B. Pan, A Review of Speckle Pattern Fabrication and Assessment for Digital Image Correlation, Experimental Mechanics 57 (8) (2017) pp. 1161-1181. doi: https ://doi .org/10.1007/s11340-017-0283-1

[3] S. Gualtieri, Novel technique for DIC speckle pattern optimization and generation, Thesis, Polotecnico di Milano (2012).

[4] D. Lecompte, A. Smits et al., Quality assessment of speckle patterns for digital image correlation, Optics and Lasers in Engineering 44 (11) (2006) pp. 1132-1145.

doi: https://doi.org/10.1016/j.optlaseng.2005.10.004

[5] A. Freddi, G. Olmi, L. Cristofolini, Experimental Stress Analysis for Materials and Structures - Stress Analysis Models for Developing Design Methodologies, Springer, New York City, 2015. doi: https://doi.org/10.1007/978-3-319-06086-6

[6] Application Note AN-1701 - Speckle Pattern Fundamentals, Correlated Solutions, 2018. 
[7] P. Reu, All about speckles: Aliasing, Experimental Techniques 38 (5) (2014) pp. 1-3.

doi: https://doi.org/10.1111/ext.12111

[8] P. Reu, All about speckles: Speckle density, Experimental Techniques 39 (3) (2015) pp. 1-2.

doi: https ://doi.org/10.1111/ext.12161

[9] P. Reu, All about speckles: Edge sharpness, Experimental Techniques 39 (2) (2015) pp. 1-2.

doi: https://doi.org/10.1111/ext.12139

[10] P. Reu, Calibration: A good calibration image, Experimental Techniques 37 (6) (2013) pp. 1-3.

doi: https ://doi.org/10.1111/ext.12059

[11] P. Reu, Points on Paint, Experimental Techniques 39 (4) (2015) pp. 1-2. doi: https://doi.org/10.1111/ext.12147

[12] P. Reu, Speckles and their relationship to the digital camera, Experimental Techniques 38 (4) (2014) pp. 1-2.

doi: https ://doi.org/10.1111/ext.12105

[13] P. Reu, Virtual Strain Gage Size Study, Experimental Techniques 39 (5) (2015) pp. 1-3.

doi: https://doi.org/10.1111/ext.12172

[14] P. Reu, All about speckles: Contrast, Experimental Techniques 39 (1) (2015) pp. 1-2.

doi: https ://doi.org/10.1111/ext.12126

[15] P. Reu, All about speckles: Speckle Size Measurement, Experimental Techniques 38 (6) (2014) pp. 1-2.

doi: https ://doi.org/10.1111/ext.12110

[16] G. Crammond, S. W. Boyd, J. M. Dulieu-Barton, Speckle pattern characterisation for high resolution digital image correlation, Applied Mechanics and Materials 70 (2011) pp. 261-266.

doi: https://doi.org/10.4028/WWW. scientific.net/AMM. 70.261 
[17] W. S. LePage, J. A. Shaw, J. A., S. H. Daly, Optimum Paint Sequence for Speckle Patterns in Digital Image Correlation, Experimental Techniques, 41(5) (2017) pp. 557-563.

doi: https ://doi .org/10.1007/s40799-017-0192-3

[18] CSI Application Note AN-525 - Speckle Pattern Fundamentals, Correlated Solutions, 2014.

[19] G. Lionello, L. Cristofolini, A practical approach to optimizing the preparation of speckle patterns for digital-image correlation, Measurement Science and Technology 25 (10) (2014) (107001).

[20] J. L. W. Carter, M. D. Uchic, M. J. Mills, Impact of Speckle Pattern Parameters on DIC Strain Resolution Calculated from In-situ SEM Experiments. In: J. Carroll, S. Daly (eds) Fracture, Fatigue, Failure, and Damage Evolution, Volume 5. Conference Proceedings of the Society for Experimental Mechanics Series. Springer, Cham, 2015, pp. 119-126. doi: https ://doi.org/10.1007/978-3-319-06977-7_16

[21] G. F. Bomarito, J. D. Hochhalter et al., Increasing accuracy and precision of digital image correlation through pattern optimization, Optics and Lasers in Engineering 91 (2017) pp. 73-85.

doi: $h t t p s: / /$ doi.org/10.1016/j .optlaseng.2016.11.005

[22] B. Pan, Z. Lu, H. Xie, Mean intensity gradient: An effective global parameter for quality assessment of the speckle patterns used in digital image correlation, Optics and Lasers in Engineering 48 (4) (2010) pp. 469-477. doi: $h$ ttps ://doi.org/10.1016/j .optlaseng . 2009.08.010

[23] P. Reu, The Art and Application of DIC; DIC: A Revolution in Experimental Mechanics, Experimental Techniques 39 (6) (2015) pp. 1-2. doi: $h$ ttps ://doi.org/10.1111/ext.12173

[24] T. Réti, Qualification of morphological similarity of microscopic tissue images by image analysis procedure, BKL - Kohászat 116 (1) (1983) pp. 1725 , in Hungarian. 